\title{
Transient behavior of surface plasmon polaritons scattered at a subwavelength groove
}

\author{
G. Lévêque* and O. J. F. Martin \\ Laboratoire de Nanophotonique et Métrologie, Ecole Polytechnique Fédérale de Lausanne, CH1015-Lausanne, Switzerland
}

\author{
J. Weiner ${ }^{\dagger}$ \\ IRSAMC/LCAR, Université Paul Sabatier, 118 route de Narbonne, 31062 Toulouse, France and IFSC/CePOF, Universidade de São \\ Paulo, Avenida Trabalhador São-Carlense, 400-CEP 13566-590, São Carlos, São Paulo, Brazil
}

(Received 4 April 2007; published 18 October 2007)

\begin{abstract}
We present a numerical study and analytical model of the optical near field diffracted in the vicinity of subwavelength grooves milled in silver surfaces. The Green's tensor approach permits the computation of the phase and amplitude dependence of the diffracted wave as a function of the groove geometry. It is shown that the field diffracted along the interface by the groove is equivalent to replacing the groove by an oscillating dipolar line source. An analytic expression is derived from the Green's function formalism, which reproduces well the asymptotic surface plasmon polariton (SPP) wave as well as the transient surface wave in the near zone close to the groove. The agreement between this model and the full simulation is very good, showing that the transient "near-zone" regime does not depend on the precise shape of the groove. Finally, it is shown that a composite diffractive evanescent wave model that includes the asymptotic SPP can describe the wavelength evolution in this transient near zone. Such a semianalytical model may be useful for the design and optimization of more elaborate photonic circuits, whose behavior in a large part will be controlled by surface waves.
\end{abstract}

DOI: 10.1103/PhysRevB.76.155418

\section{INTRODUCTION}

The analysis of light diffraction and transmission through a slit has a long history in physical optics. ${ }^{1,2}$ As discussed by Kowarz, ${ }^{3,4}$ the analysis can be separated into two problems: the boundary value problem and the propagation problem. The boundary value problem concerns the determination of the field immediately at the output plane, and interest is usually concentrated on the boundary values in the vicinity of the slit. The propagation problem involves determination of the field at points in the half-space beyond the output plane in the near and far fields. Recent interest in light transmission through subwavelength periodic structures with subwavelength pitch $^{5,6}$ has stimulated some experimental ${ }^{7-11}$ and many theoretical studies ${ }^{12-23}$ with the aim of better understanding the nature of the light field at the surface (the boundary value problem) and the influence of this surface field on light transmission (the propagation problem).

We report here numerical simulations of single groove and slit-groove structures using a Green's tensor method to solve the Maxwell field equations near the subwavelength structures on the metal/free space interface. The simulations are compared with recent experimental results on single slitgroove structures, ${ }^{8-10}$ and essentially confirm the observed amplitude and phase evolution of the scattered waves as a function of groove geometry and groove-slit distance. We then show that the results of the full numerical simulation can be recovered by replacing the groove structure with a simple oscillating dipole line source and again applying the Green's tensor method to find the near and far fields along the surface. This oscillating dipole picture is consistent with recent charge and field distributions at metal-slit and metalgroove boundaries found numerically by a finite-difference time domain (FDTD) technique ${ }^{20}$ and permits calculation of both the propagating and evanescent contributions to the scattered field. It is also in the spirit of earlier work investigating dipolar interaction of molecules on surfaces. ${ }^{13}$ Finally,
PACS number(s): 42.25.Fx, 73.20.Mf, 78.67.-n

we also present a simple analytic aperture-in-opaque-screen model, in the same vein as earlier models, ${ }^{3,4,7}$ but with a boundary condition that posits the surface plasmon polariton (SPP) mode at the metal/free space interface. While comparison of the Green's tensor simulations to the analytic model helps to physically interpret the numeric results in terms of composite surface-wave modes, the oscillating dipole picture provides deeper insight into the essential physics of surfacewave generation at the groove while overcoming the limitations of any fixed-boundary-condition model.

\section{NUMERICAL SIMULATIONS}

The numerical simulations are performed with the Green's tensor method. ${ }^{24,25}$ This method is very convenient for the study of finite-size, two-dimensional (2D) or threedimensional (3D) objects embedded in a multilayered background. It relies on the resolution of the LippmannSchwinger equation of the electric field,

$$
\mathbf{E}(\mathbf{r})=\mathbf{E}^{0}(\mathbf{r})+k_{0}^{2} \int_{V} d \mathbf{r}^{\prime} \mathbf{G}\left(\mathbf{r}, \mathbf{r}^{\prime}\right) \Delta \epsilon\left(\mathbf{r}^{\prime}\right) \mathbf{E}\left(\mathbf{r}^{\prime}\right),
$$

where $\mathbf{G}\left(\mathbf{r}, \mathbf{r}^{\prime}\right)$ is the Green's tensor associated with the stratified background, $k_{0}^{2}=\omega^{2} \epsilon_{0} \mu_{0}$ is the square of the wave propagation parameter, and $\Delta \epsilon(\mathbf{r})$ is the "dielectric contrast," the relative permittivity difference between the scatterer of volume $V$ and the adjacent layer. The Green's tensor itself is the solution of a vector wave equation with a point dipole source,

$$
\boldsymbol{\nabla} \times \boldsymbol{\nabla} \times \mathbf{G}\left(\mathbf{r}, \mathbf{r}^{\prime}\right)-k_{0}^{2} \epsilon \mathbf{G}\left(\mathbf{r}, \mathbf{r}^{\prime}\right)=\mathbf{1} \delta\left(\mathbf{r}-\mathbf{r}^{\prime}\right),
$$

where $\mathbf{1}$ is the unit tensor. The 3D Green's tensor represents the electric field at $\mathbf{r}$ produced by three orthogonal unit dipoles located at $\mathbf{r}^{\prime}$ in a layer of dielectric constant $\epsilon$. An advantageous distinguishing property of the Green's tensor 


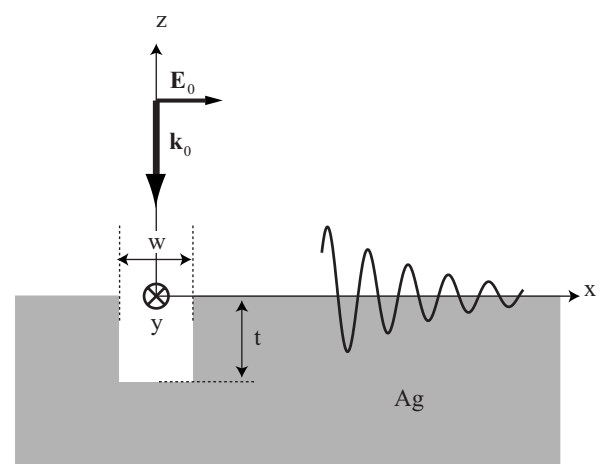

FIG. 1. Geometry of the single groove system.

method is that only the objects of interest need be discretized. The boundary conditions at infinity are included in the Green's tensor of the multilayered background. In the present case, the calculation takes a very short time because of the small size (some tens of nanometers) of the groove and slit. Details and extensive references concerning this method can be found in a recent review. ${ }^{26}$

The system initially studied is shown in Fig. 1. It is an empty groove milled into a metallic substrate, with the groove profile along $x$ and extending invariantly along $y$. Outside the groove, the metal/free space interface lies in the $0 x y$ plane with $z$ axis in the vertical direction. The rectangular section of the groove is characterized by width $w$ and depth $t$. The incident free-space electromagnetic plane wave, with $E$-field polarized along $x$, impinges on the groove and interface at normal incidence. Because of the reduced dimensionality of the problem, all scattered waves, propagating and evanescent, are restricted to the $0 x z$ plane.

The electric field is calculated along the line $z=0^{+}, x>0$. All simulations are performed at the free-space wavelength $\lambda_{0}=852 \mathrm{~nm}$. In order to compare these results with previously published studies, ${ }^{8,10,23}$ the relative permittivity (dielectric constant) of silver is taken to be $\epsilon_{m}=-33.22+1.17 i$. The corresponding propagation length against absorption is $680 \mu \mathrm{m}$, quite long because the imaginary term in the dielectric constant is small for silver at this wavelength. The results of the calculation are plotted in Fig. 2. The principal plot in Fig. 2 shows the evolution of the effective index $n_{\text {eff }}$ $=\lambda(x) / \lambda_{0}$ as a function of the distance from the center of the groove, for both $E_{x}$ and $E_{z}$ components. The dotted line indicates the effective index of the SPP guided wave,

$$
\lambda_{\text {spp }}=\lambda_{0} \mathfrak{R}\left[\left(\frac{1+\epsilon_{m}}{\epsilon_{m}}\right)^{1 / 2}\right]=839.1 \mathrm{~nm}
$$

for the silver/free space interface. The variation of the effective index is different for the two components, but both curves converge rapidly after $3 \mu \mathrm{m}$. In both cases, the effective index is larger than $n_{\mathrm{spp}}$ out to $\sim 10 \mu \mathrm{m}$, but for greater distances, it converges to the expected $n_{\text {spp }}$. The results of the simulation are consistent with the measurements of Ref. 8, which reported a value of $n_{\text {surf }}=1.04 \pm 0.01$ over a distance of $\sim 6 \mu \mathrm{m}$. They are also consistent with recent FDTD simulations on silver surfaces ${ }^{10}$ as well as similar measurements and simulations on gold surfaces. ${ }^{11}$ The inset of Fig. 2 shows

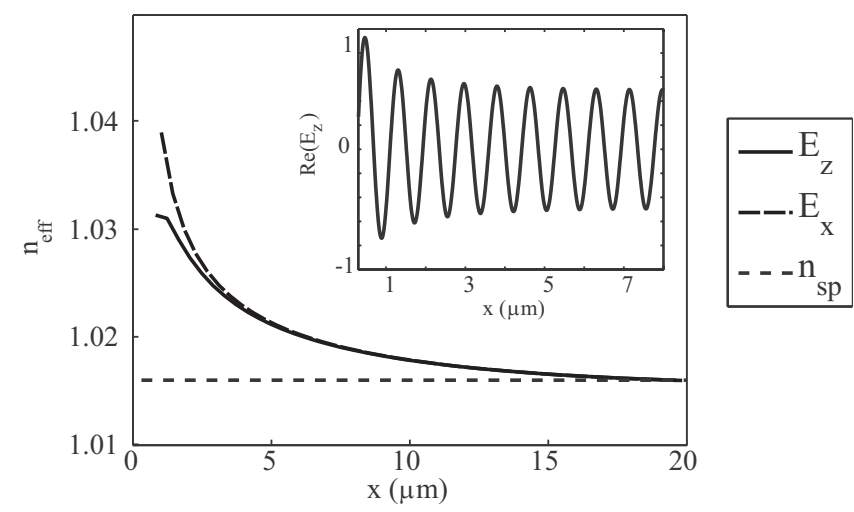

FIG. 2. Evolution of the $E_{z}$ (solid curve) and $E_{x}$ (dashed curve) effective index $n_{\text {eff }}(x)=\lambda(x) / \lambda_{0}$ with the distance from the center of the groove. The groove dimensions are $t=100 \mathrm{~nm}$ and $w=100 \mathrm{~nm}$. Inset: Evolution of the real part of $E_{z}$ with $x$, along the $z=0^{+}$line.

the $z$ component of the electric field diffracted by the groove along the interface. We can clearly identify two regimes. The first extends out to $\simeq 3 \mu \mathrm{m}$ along $x$ and is characterized by a relatively rapid decay of the amplitude. For further distances, the amplitude decreases much more slowly (due to absorption) and appears constant over the displayed range. This two-step evolution is characteristic of a transient regime within the first few micrometers from the groove. Since the incident wave is transverse magnetic (TM) polarized ( $E$-field parallel to $x$ ), $E_{z}$ belongs only to the scattered field and does not interfere with the incident wave. For this reason, the mean value of the real part of $E_{z}$ in the total field along the interface must be zero. This is not the case for the $x$ component, as the incident field is polarized along the $x$ direction.

The simulations also show that the phase and the amplitude of the diffracted wave are sensitive to the groove dimensions, as reported in experiments. ${ }^{9}$ In Figs. 3(a) and 3(b) are plotted the amplitude and phase evolution of the surface wave, at large distance $(18 \mu \mathrm{m})$. The phase $\varphi_{\text {int }}$ has been determined by comparison with a cosine function representing the SPP guided surface wave. It corresponds to the asymptotic phase of the diffracted wave, called the "intrinsic phase" in Ref. 9. The evolution of the scattered wave phase and amplitude as a function of groove depth is typical of a resonant phenomenon. Here, the resonance concerns standing-wave modes created inside the groove. The $w$ and $t$ dimensions of the groove may be varied so as to produce a cavity that resonates when excited by an incident surface wave. At resonance, a vertical standing mode dominates the field distribution inside the groove. Because of the boundary conditions, the electric field must be almost null at the bottom of the groove. Near a resonance, the phase of the diffracted wave varies rapidly and passes through an inflection point, while the amplitude reaches a maximum. As can be observed in Fig. 3, the amplitude is maximal when the phase is almost $\pi$. The experimental results ${ }^{9}$ reported an intrinsic phase value of $\pi / 2$ at the resonance groove depth. This difference of $\pi / 2$ between the experiment and the simulation arises from the fact that $x$ and $z$ components of the surface wave $E$-field oscillate in quadrature. The experiment essentially measures the intrinsic phase difference in a far-field 


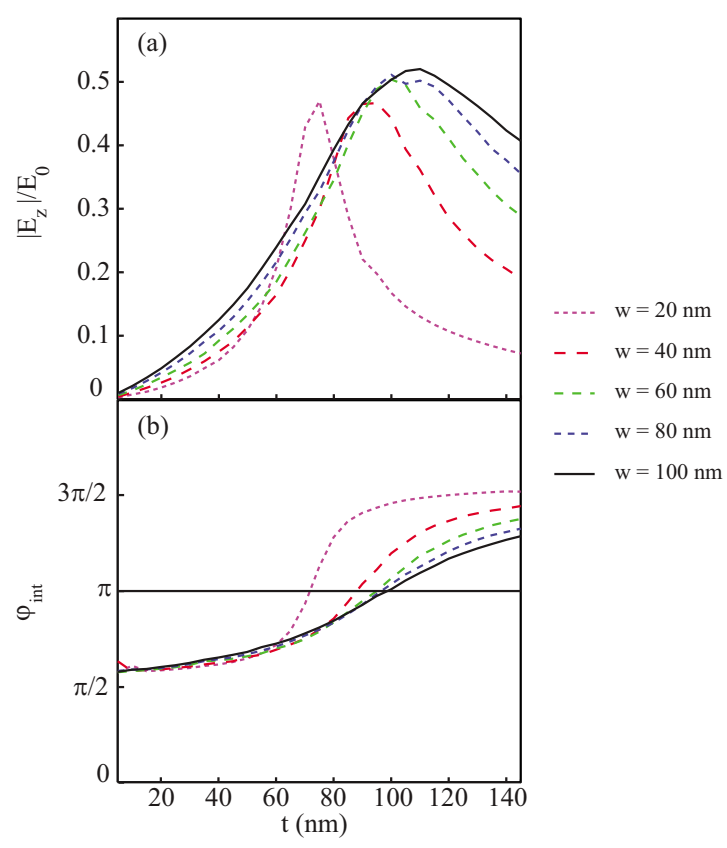

FIG. 3. (Color online) (a) Evolution of the amplitude of the $z$ component of the electric field, $18 \mu \mathrm{m}$ away from the groove. (b) Evolution of the intrinsic phase $\varphi_{\text {int }}$ of the $z$ component of the electric field diffracted by the groove.

interference pattern between two oscillating dipoles oriented along $x$ : one localized at the corners of a slit and the other localized at the groove (see inset of Fig. 4). Thus, the experiment is sensitive to the intrinsic phase of the $x$ component of the surface $E$-field, while the simulation calculates the intrinsic phase of the $z$ component. After taking this quadrature phase difference into account, we see that the simulations are consistent with the measurements. The groove depth for which the resonance occurs increases with the width. For a width of $w=100 \mathrm{~nm}$, the simulations yield an optimal depth $t \approx 110 \mathrm{~nm}$. For shallow depths, some tens of nanometers, the amplitude and phase depend weakly on the width. At

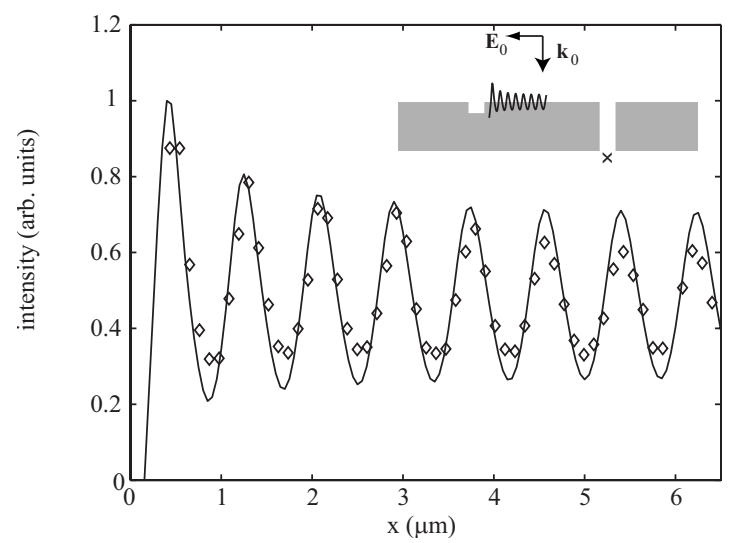

FIG. 4. Comparison between the experimental results of Ref. 8 and the numerical simulations performed with a groove of width $w=100$ and depth of $t=120 \mathrm{~nm}$. The silver slab is $400 \mathrm{~nm}$ thick, and the slit is $100 \mathrm{~nm}$ wide. The electric-field intensity was computed at the exit of the slit (black cross).

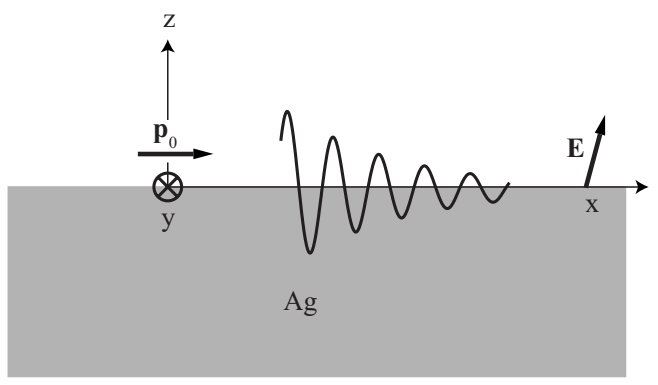

FIG. 5. The source is a dipole line located along the $y$ axis at $z=0^{+}$, and oriented parallel to the $x$ axis. The field is computed along $\left(x, z=0^{+}\right)$.

greater depths, the amplitude becomes quite sensitive to the groove width, but the phase does not change dramatically for widths $w>40 \mathrm{~nm}$. Clearly, the phase and the amplitude of the diffracted wave resonance is very sensitive to the groove geometry. As the absolute groove depth is difficult to determine experimentally, the simulation results for ideal geometries may differ somewhat from the nominal parameters of fabricated structures.

In the case of the slit-groove experiments reported in Ref. 8 , the Green's tensor simulations produced the best agreement with the experimental points by considering a depth of $t=120 \mathrm{~nm}$, rather than the nominal experimental depth of $100 \mathrm{~nm}$. A comparison between the simulation and measurement is plotted in Fig. 4; only the initial amplitude has been normalized to the experimental curve. Although the experimental intensity derives from a far-field interference fringe and the simulated field is evaluated at the output-side plane, it is legitimate to compare the two curves because the farfield signal is proportional to the calculated field intensity at the output-side slit exit. We note that the same slit-groove calculation performed with $t=100 \mathrm{~nm}$ is in excellent agreement with the simulation of Lalanne and Hugonin ${ }^{23}$ using an entirely different simulation technique.

\section{FIELD SCATTERED BY A DIPOLE ALONG THE INTERFACE}

In this section, we consider the 2D field radiated by a line dipole $\mathbf{p}_{0}$ (rather than the 3D field radiated by a point dipole) located just above the metal/free space interface, as indicated in Fig. 5. The choice of placing the dipole just above the surface may seem arbitrary, but it is shown in the Appendix that placing the dipole just under the interface leads to the same conclusions. The dipole is aligned parallel to the $x$ axis, consistent with the previous groove calculations of Sec. II. For the same reasons discussed there, we only calculate the expression of the $z$ component of the electric field. The dipole oscillation wavelength is $852 \mathrm{~nm}$. We will use the Green's tensor formalism to extract a simple expression for the field just above the interface.

The field radiated by the dipole at a point just above the interface at a distance $x$ is given by the equation

$$
\mathbf{E}\left(\mathbf{r}, \mathbf{r}_{0}\right)=k_{0}^{2}\left[\mathbf{G}_{0}\left(\mathbf{r}, \mathbf{r}_{0}\right)+\mathbf{G}_{S}\left(\mathbf{r}, \mathbf{r}_{0}\right)\right] \mathbf{p}_{0},
$$

with $\mathbf{r}(x, z)=\left(x, 0^{+}\right)$and $\mathbf{r}_{0}=\left(0,0^{+}\right)$. We denote the couple $\left(\mathbf{r}, \mathbf{r}_{0}\right)$ as $(x, 0)$. The $3 \times 3$ tensors $\mathbf{G}_{0}$ and $\mathbf{G}_{S}$ are the dyadic 
Green's functions associated with free space and the metal/ free space surface at the considered wavelength. Thus, the first term represents the field directly radiated by the dipole to the observation point through free space. The second term represents the field radiated to the observation point after reflection from the surface. The observation point is displaced along $x$, on a line just above and parallel to the surface, running through the dipole. Due to symmetry of the dipole radiation pattern, the $z$ component of the directly radiated term, $\mathbf{G}_{0}$, along the line of observation points is 0 , and we have

$$
\begin{aligned}
E_{z}(x) & =k_{0}^{2}\left[\mathbf{G}_{0}(x, 0) \mathbf{p}_{0}+\mathbf{G}_{S}(x, 0) \mathbf{p}_{0}\right] \cdot \mathbf{e}_{z} \\
& =k_{0}^{2}\left[\mathbf{G}_{S}(x, 0) \mathbf{p}_{0}\right] \cdot \mathbf{e}_{z}=k_{0}^{2} G_{S}^{z x}(x, 0) p_{0},
\end{aligned}
$$

where $\mathbf{e}_{z}$ is the unit vector of the $z$ axis and $G_{S}^{z x}$ is the $z x$ component of the surface Green's function, i.e., the $z$ component of the Green's function produced by a dipole aligned parallel to $x$.

Although there exist approximate expressions for $x$ small compared to the wavelength (electrostatic approximation), these expressions are not appropriate here because the line of observation points extends far beyond a wavelength. The exact expression of the surface Green's function cannot be written in closed form in direct space, but we can find an expression susceptible to numerical evaluation by standard methods. The Green's tensor is analytically defined in the half Fourier space $(q, z)$, where $q$ is the spatial frequency parallel to the $x$ axis. The general expression can be found in Ref. 25. The $G_{S}^{z x}$ component is given by

$$
G_{S}^{z x}\left(\mathbf{r}, \mathbf{r}^{\prime}\right)=-\frac{i}{4 \pi k_{0}^{2}} \int_{-\infty}^{+\infty} d q q R(q) e^{i q x}
$$

where $R$ is the Fresnel reflection coefficient for TM polarization and $\epsilon(\omega)$ is the metal dielectric constant:

$$
R(q)=\frac{\sqrt{\epsilon(\omega) k_{0}^{2}-q^{2}}-\epsilon(\omega) \sqrt{k_{0}^{2}-q^{2}}}{\sqrt{\epsilon(\omega) k_{0}^{2}-q^{2}}+\epsilon(\omega) \sqrt{k_{0}^{2}-q^{2}}} .
$$

Then, Eq. (3) becomes

$$
E_{z}(x)=-\frac{i p_{0}}{4 \pi k_{0}^{2}} \int_{-\infty}^{+\infty} d q q R(q) e^{i q x} .
$$

In order to interpret this last equation, consider the value of the integral without the reflection coefficient:

$$
I(x)=\int_{-\infty}^{+\infty} d q q e^{i q x} .
$$

This function $I$ is the derivative of the function $J$ :

$$
J(x)=-i \int_{-\infty}^{+\infty} d q e^{i q x}
$$

proportional to a Dirac delta function located at $x=0$. So $I$ is the derivative of a Dirac delta function that, in fact, represents a point dipole located at $x=0$ : the $z$ component of the electric field in the direction of the dipole is 0 everywhere, except at two points infinitely close where it is not defined.

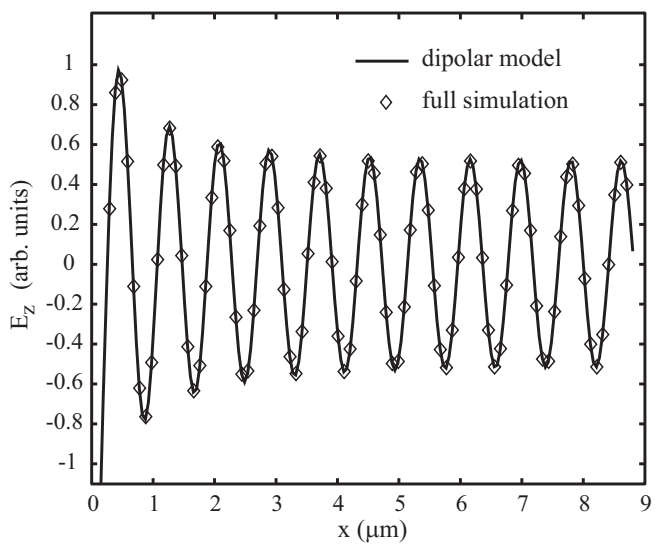

FIG. 6. Comparison between the field obtained from Eq. (5) and with the full Green's tensor numeric simulation.

Thus, Eq. (5) simply states that plane waves diffracted by the dipole in the $x$ direction are reflected by the surface with a factor given by the Fresnel reflection coefficient.

Because $q R(q)$ is an odd function of $q$ :

$$
E_{z}(x)=\frac{p_{0}}{2 \pi k_{0}^{2}} \int_{0}^{+\infty} d q q R(q) \sin q x .
$$

This integral cannot be expressed in closed form, but can be computed by conventional numerical techniques. In Fig. 6 are compared the $z$ components of the electric field computed with the groove simulation (for $t=w=100 \mathrm{~nm}$ ) and with Eq. (6). The two curves, after proper normalization, agree very well. It might appear surprising that the phase of the dipolar model does not need to be adjusted compared to the groove calculation, but Fig. 3 shows that the phase of the wave diffracted by the groove is precisely $\pi$ for this groove geometry. The overall conclusion is that it is the accumulation of oscillating charges at the corners of the groove, rather than details of the groove profile itself, that plays a key role in the global shape of the diffracted wave a few hundreds of nanometers away from the groove center. The field is essentially the field diffracted by a dipole placed near the surface; its structure is determined by the fact that the source has a broadband spatial frequency spectrum and that the surface supports a long-lived, guided SPP mode.

An interesting point is the role of constituent propagative and evanescent modes in the creation of the surface wave. In particular, the composite diffracted evanescent wave (CDEW) model, $^{7}$ previously invoked to interpret similar phenomena, considers explicitly only the evanescent part. The two contributions are difficult to extract from full numerical simulations, but can be easily carried out with the dipole approach. The scattered field of Eq. (6) is separated in its propagative and evanescent components:

$$
E_{z}(x)=E_{z}^{p r}(x)+E_{z}^{e v}(x),
$$

with

$$
E_{z}^{p r}(x)=\frac{p_{0}}{2 \pi k_{0}^{2}} \int_{0}^{k_{0}} d q q R(q) \sin q x,
$$




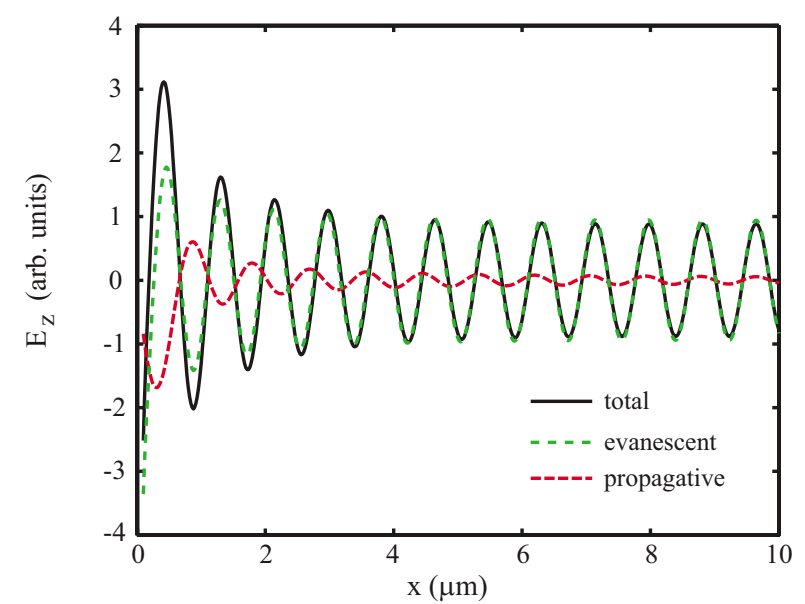

FIG. 7. (Color online) Contribution to the field of the radiative and evanescent components to the total wave calculated from Eq. (7).

$$
E_{z}^{e v}(x)=\frac{p_{0}}{2 \pi k_{0}^{2}} \int_{k_{0}}^{+\infty} d q q R(q) \sin q x .
$$

Figure 7 compares the real part of these two contributions. The propagative term represents a substantial fraction, its amplitude being around $50 \%$ of the total wave a few hundreds of nanometers from the dipole location. However, the amplitude of the propagative component decreases much faster than the evanescent term, and the wavelength of the propagative part is clearly longer than the wavelength of the evanescent component. The reason is that for all evanescent modes, including the SPP mode, $q>k_{0}, \lambda<\lambda_{0}$, and $n_{\text {eff }}>1$. For the propagative modes, $q \leqslant k_{0}, \lambda \geqslant \lambda_{0}$, and $n_{\text {eff }} \leqslant 1$.

These trends appear clearly in Fig. 8, which represents the evolution with distance from the dipole of the effective indices of refraction for the different contributions. The index of the evanescent part is larger than the SPP index and de-

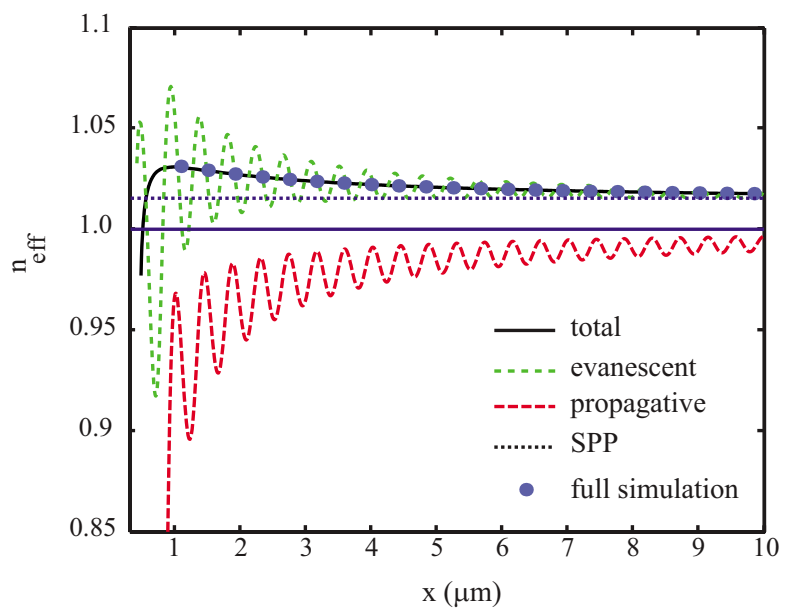

FIG. 8. (Color online) Contribution to the effective index of the radiative and evanescent components to the total wave, and comparison with the effective index calculated with the groove full simulation. creases to this value asymptotically, but the index of the propagative part increases with distance and tends toward $n$ $=1$. This is a consequence of the fact that the propagative wave is dominated by grazing plane waves, for which the reflection coefficient is almost equal to 1 , which implies $q$ $=k_{0}$ and, therefore, $n=1$. When these two contributions are summed, the effective index follows the curve computed from the groove simulation and is in good agreement with measurement. ${ }^{8}$

\section{ANALYTICAL MODEL}

The dipole-on-surface model can be further simplified in order to express the diffracted wave in closed form. In the following, we present a simple opaque-screen analytic model, similar to that of Kowarz ${ }^{4}$ and to the CDEW model of Lezec and Thio, ${ }^{7}$ but in which the SPP wave is introduced as a boundary condition on the surface. In the Kowarz and CDEW models, the evanescent part of the diffracted wave is computed assuming the presence of a slit in an infinitely thin opaque screen. With these assumptions, the amplitude of the evanescent wave along the interface is given by

$$
\begin{aligned}
E(x)= & \int_{|q|>\left|k_{0}\right|}^{\infty} \frac{\sin (q w / 2)}{q} e^{i q x} d q=-\frac{E_{0}}{\pi}\left[\operatorname{Si}\left(k_{0}\left(x+\frac{w}{2}\right)\right)\right. \\
& \left.-\operatorname{Si}\left(k_{0}\left(x-\frac{w}{2}\right)\right)\right], \quad|x|>\frac{w}{2}
\end{aligned}
$$

where $\mathrm{Si}$ is the sine integral defined as

$$
\operatorname{Si}(\alpha)=\int_{0}^{\alpha} \frac{\sin t}{t} d t .
$$

The interpretation is straightforward: the slit diffracts the incident wave into a sum of evanescent waves of spatial frequency $q$, whose amplitudes are weighted by the Fourier amplitudes of the slit. The Fourier spectrum of the slit is a cardinal sine (sinc) function. Moreover, when $x \rightarrow \infty$, the solution of Eq. (8) is correctly approximated by

$$
E(x) \approx \frac{E_{0}}{\pi} \frac{d}{x} \cos \left(k_{0} x+\frac{\pi}{2}\right) .
$$

Neither the Kowarz nor the CDEW model, however, reproduce correctly the result of Ref. 8. One of the reasons is that the finite conductivity of the screen is not included. If we consider again a TM-polarized wave incident on the groove, the SPP mode of complex wave vector $q_{\mathrm{spp}}$ is excited along the interface. In that case, the corresponding wave vector is created by the diffraction of the incident wave. The dispersion relation of the SPP guided mode, $q_{\mathrm{spp}}=k_{0} \sqrt{\epsilon_{m} /\left(\epsilon_{m}+1\right)}$, can be retrieved by calculating the pole of the reflection coefficient of the metal/free space interface $R(q)$. At $\lambda_{0}$ $=852 \mathrm{~nm}$ and the value of $\epsilon$ for silver, $q_{\mathrm{spp}}=(1.014+i 2$ $\left.\times 10^{-4}\right) k_{0}$. For an evanescent wave whose wave vector is near $q_{\text {spp }}$, the reflection coefficient can be approximated by $A /\left(q-q_{\text {spp }}\right)$, where $A$ is a constant. We can say that the incident wave impinging on the groove is at first diffracted, with amplitude corresponding to the Fourier spectrum of the 
groove, and is then reflected along the metallic interface with a coefficient $R(q)$. Hence, an approximate expression of the evanescent wave propagating along the interface is obtained by replacing Eq. (8) with

$$
\begin{aligned}
E(x)= & \int_{-\infty}^{-k_{0}} \frac{\sin (q w / 2)}{q} \frac{1}{q+q_{\mathrm{spp}}} e^{i q x} d q \\
& +\int_{+k_{0}}^{\infty} \frac{\sin (q w / 2)}{q} \frac{1}{q-q_{\mathrm{spp}}} e^{i q x} d q .
\end{aligned}
$$

Here, two poles must be inserted because the SPP wave is excited in both $\pm x$ directions. This expression can be simplified using the fact that the width of the groove Fourier spectrum is of the order of $1 / w$, whereas the width of the "spectral line" of the Fourier SPP mode is of the order of $\Im\left(q_{\mathrm{spp}}\right)$, a thousand times narrower than the Fourier spectrum of the groove. Hence, in $q$ space, the groove structure is essentially a constant over the width of the SPP response. Changing the width of the groove will only modify the amplitude of the plane waves of wave vector $q \approx q_{\mathrm{spp}}$, and thus, the amplitude of the diffracted wave. Hence, we have

$$
E(x) \approx \int_{-\infty}^{-k_{0}} \frac{1}{q+q_{\mathrm{spp}}} e^{i q x} d q+\int_{+k_{0}}^{\infty} \frac{1}{q-q_{\mathrm{spp}}} e^{i q x} d q .
$$

For $x>w / 2$, this expression reads

$$
E(x)=2 \pi e^{i q_{\text {spp }}{ }^{x}} G(x),
$$

with

$$
G(x)=i-\frac{\operatorname{Ei}\left(i\left(k_{0}-q_{\mathrm{spp}}\right) x\right)}{2 \pi}+\frac{\operatorname{Ei}\left(i\left(-k_{0}-q_{\mathrm{spp}}\right) x\right)}{2 \pi} .
$$

The function Ei is called exponential integral and is defined by

$$
\operatorname{Ei}(z)=-\int_{-z}^{\infty} \frac{e^{-t}}{t} d t
$$

It appears that the amplitude of the SPP wave propagating along $x, \exp \left(i q_{\mathrm{spp}} x\right)$, is multiplied by an envelope of complex value $G(x)$. This function is represented in the complex plane for typical parameters of SPP wave vector in Fig. 9(a). The low values of $x$ correspond to the right of the curve. When $x$ goes toward infinity, $G(x)$ whirls toward $z_{\text {lim }}=i$. The strong oscillation at the beginning of the curve is due to a beating between $\operatorname{Ei}\left(-q_{\mathrm{spp}}\right)$ and $\operatorname{Ei}\left(+q_{\mathrm{spp}}\right)$ terms. As this function has a varying phase, it will affect the wavelength of the surface wave. Figure 10 compares the evolution of the surface index for $x$ and $z$ components using the index computed from the previous formula. This analytical model predicts the same result as the numerical and dipole approaches: the effective index of the wave generated near the groove is greater than $n_{\text {spp }}$, but decreases and converges toward the expected SPP value within a range of about $10 \mu \mathrm{m}$. In fact, the effective index oscillates slightly around $n_{\text {spp }}$ at larger distances. There is good qualitative agreement with the dipole model, because the evanescent waves play the main role in the creation of the surface wave. The fact that the reflection coefficient is replaced by a simple pole in $q_{\mathrm{spp}}$ changes essentially the time

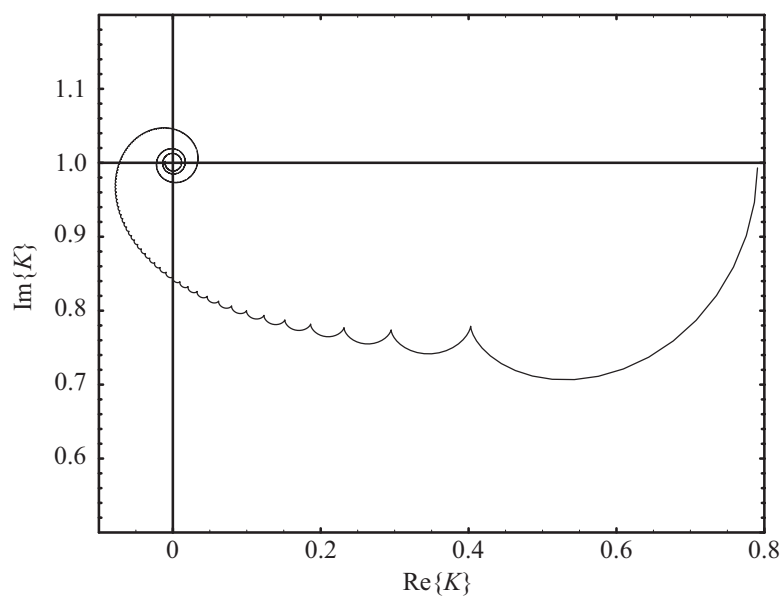

FIG. 9. Parametric curve representing the function $i-[\operatorname{Ei}[i(1$ $-\alpha) t] / 2 \pi]+[\operatorname{Ei}[-i(1+\alpha) t] / 2 \pi]$ in the complex plane. In this example, $\alpha=q_{\mathrm{spp}} / k 0=1.02$.

evolution of the wave amplitude (not shown). However, the wavelength evolution is correct. The effective index is overestimated in the first micrometers because the radiative part is not taken into account.

\section{SUMMARY}

We have studied in detail the structure of the wave diffracted by a groove or slit milled in a metallic surface, illuminated by a monochromatic plane wave. First, the Green's tensor method has been used to analyze the amplitude, phase, and frequency behavior of the surface wave in the vicinity of the groove. In this zone, the surface wave has a transient regime characterized by a rapid variation of the amplitude within the first $2-3 \mu \mathrm{m}$, and an increase of the surface wavelength up to the value of the SPP wavelength in the first $15 \mu \mathrm{m}$. The phase and the amplitude of the scattered wave depend strongly on the groove geometry, as the incident wave excites an "organ-pipe" mode inside the groove. Best agreement with the experimental results of Gay et al. ${ }^{8}$ is

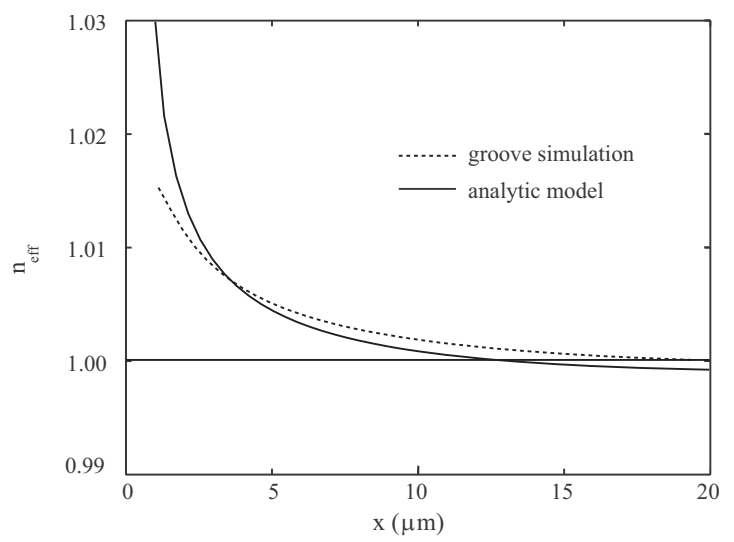

FIG. 10. Comparison between the $E_{z}$ effective index and the effective index computed from the analytical model. The groove's dimensions are $t=100 \mathrm{~nm}$ and $w=100 \mathrm{~nm}$. 
obtained assuming a somewhat deeper groove $(120 \mathrm{~nm})$ than in the experiment $(100 \mathrm{~nm})$. This value is within the uncertainty of the actual milled depth using focused ion beam fabrication. We have also presented a simplified model in which the surface wave is excited by a line dipole parallel to and just above a flat surface without groove. This approach permits the extraction of an analytical expression for the $z$ component of the electric field along the interface. The agreement between this model and the full simulation is very good, showing that the transient "near-zone" regime does not depend on the precise shape of the groove. Indeed, the details of groove depth and width only influence the amplitude and the phase of the generated wave. The overall form results from a line dipole source with a broad $q$ spectrum interacting with a surface that supports a guided mode. Moreover, we have studied the influence on the wave structure of the propagative and evanescent contributions. The propagative waves contribute importantly in the first few micrometers from the source, but their amplitude decay is faster and their wavelength is longer than the evanescent contribution. The wavelength of the propagative contribution decreases with the distance down to the excitation wavelength, whereas the effective wavelength of the evanescent contribution increases up to the SPP effective wavelength. Finally, we have studied a simplified model of the diffraction process, in which the reflection coefficient is replaced by a pole located at the SPP wave vector in $q$ space. The scattered field can then be expressed in closed form. This "minimal model" correctly reproduces the SPP excitation and the wavelength evolution with distance. Such a semianalytical model may be useful for the design and optimization of more elaborate photonic circuits, whose behavior in a large part will be controlled by surface waves.

\section{ACKNOWLEDGMENTS}

G.L. and O.J.F.M. acknowledge funding from the IST Network of Excellence Plasmonanodevices (FP6-2002-IST1-507879). J.W. acknowledges support from the Ministère délégué à l'Enseignement supérieur et à la Recherche under the program ACI-"Nanosciences-Nanotechnologies," the Région Midi-Pyrénées (SFC/CR 02/22), and FASTNet (HPRNCT-2002-00304) EU Research Training Network. Support from the research foundation FAPESP of the State of São Paulo, Brazil, is also gratefully acknowledged.

\section{APPENDIX}

The purpose of this appendix is to show that the location of the dipole, just above or below the metal/free space interface, is independent of the results obtained in Sec. III. We begin with Eq. (6):

$$
E_{z}(x)=\frac{p_{0}}{2 \pi k_{0}^{2}} \int_{0}^{+\infty} d q q[R(q)-R(\infty)+R(\infty)] \sin q x .
$$

The integrand diverges when $q \rightarrow+\infty$. However, the integral is defined for $x>0$. We can write Eq. (A1) as

$$
E_{z}(x)=\frac{p_{0}}{2 \pi k_{0}^{2}}\left[\int d q q \widetilde{R}(q) \sin q x+R(\infty) \int d q q \sin q x\right],
$$

where

$$
R(\infty)=\frac{1-\epsilon(\omega)}{1+\epsilon(\omega)} \quad \text { and } \quad \widetilde{R} \equiv R(q)-R(\infty)
$$

so that from Eq. (4),

$$
\tilde{R}(q)=\frac{2 \epsilon(\omega)}{1+\epsilon(\omega)} \frac{\sqrt{\epsilon(\omega)-q^{2}}-\sqrt{1-q^{2}}}{\sqrt{\epsilon(\omega)-q^{2}}+\epsilon(\omega) \sqrt{1-q^{2}}} .
$$

The first part of Eq. (A2) converges because

$$
q \widetilde{R}(q) \underset{q \rightarrow+\infty}{\longrightarrow} \frac{\gamma}{q} \text { with } \quad \gamma \in \mathbf{C} .
$$

The second part is equal to the derivative of a Dirac function. Hence,

$$
E_{z}(x)=\frac{p_{0}}{2 \pi k_{0}^{2}} \int d q q \widetilde{R}(q) \sin q x+\alpha \delta^{\prime}(x) .
$$

The right term of the sum is only a term located at $x=0$. For numerical integration, it is more convenient to use this last expression.

If the dipole is located just under the interface,

$$
E_{z}^{-}(x)=\frac{p_{0}}{2 \pi k_{0}^{2}} \int_{0}^{+\infty} d q q T(q) \sin q x,
$$

with

$$
T(q)=\frac{2 \sqrt{\epsilon(\omega) k_{0}^{2}-q^{2}}}{\sqrt{\epsilon(\omega) k_{0}^{2}-q^{2}}+\epsilon(\omega) \sqrt{k_{0}^{2}-q^{2}}} .
$$

Here,

$$
T(q) \underset{q \rightarrow+\infty}{\longrightarrow} T(\infty)=\frac{2}{1+\epsilon(\omega)} .
$$

Hence,

$$
\begin{aligned}
E_{z}^{-}(x) & =\frac{p_{0}}{2 \pi k_{0}^{2}}\left\{\int_{0}^{+\infty} d q q \widetilde{T}(q) \sin q x+T(\infty) \int d q q \sin q x\right\} \\
& =\frac{p_{0}}{2 \pi k_{0}^{2}} \int d q q \widetilde{T}(q) \sin q x+\beta \delta^{\prime}(x),
\end{aligned}
$$

with

$$
\widetilde{T}(q)=\widetilde{R}(q) .
$$

Hence, the field diffracted by two dipoles located just above or just under the vaccum/metal interface differs only in $x$ $=0$. 
*gaetan.leveque@epfl.ch

†jweiner@irsamc.ups-tlse.fr

${ }^{1}$ C. J. Bouwkamp, Rep. Prog. Phys. 17, 35 (1954).

${ }^{2}$ M. Born and E. Wolf, Principles of Optics, 6th ed. (Pergamon, New York, 1980).

${ }^{3}$ M. W. Kowarz, Ph.D. thesis, University of Rochester, 1995.

${ }^{4}$ M. W. Kowarz, Appl. Opt. 34, 3055 (1995).

${ }^{5}$ T. W. Ebbesen, H. J. Lezec, H. F. Ghaemi, T. Thio, and H. J. Wolff, Nature (London) 391, 667 (1998).

${ }^{6}$ W. L. Barnes, A. Dereux, and T. W. Ebbesen, Nature (London) 424, 824 (2003).

${ }^{7}$ H. J. Lezec and T. Thio, Opt. Express 12, 3629 (2004).

${ }^{8}$ G. Gay, O. Alloschery, B. Viaris de Lesegno, C. O’Dwyer, J. Weiner, and H. J. Lezec, Nat. Phys. 2, 262 (2006).

${ }^{9}$ G. Gay, O. Alloschery, B. Viaris de Lesegno, J. Weiner, and H. J. Lezec, Phys. Rev. Lett. 96, 213901 (2006).

${ }^{10}$ G. Gay, O. Alloschery, J. Weiner, H. J. Lezec, C. O’Dwyer, M. Sukharev, and T. Seideman, Phys. Rev. E 75, 016612 (2007).

${ }^{11}$ F. Kalkum, G. Gay, O. Alloschery, J. Weiner, H. J. Lezec, Y. Xie, and M. Mansuripur, Opt. Express 15, 2613 (2007).

${ }^{12}$ Much of the early theoretical work on light diffracting from periodic structures has been documented in R. Petit, Electromagnetic Theory of Gratings, Topic in Current Physics Vol. 22
(Springer-Verlag, Berlin, 1980).

${ }^{13}$ G. W. Ford and W. H. Weber, Phys. Rep. 113, 195 (1984).

${ }^{14}$ M. J. Treacy, Appl. Phys. Lett. 75, 606 (1999).

${ }^{15}$ M. M. J. Treacy, Phys. Rev. B 66, 195105 (2002).

${ }^{16}$ Q. Cao and P. Lalanne, Phys. Rev. Lett. 88, 057403 (2002).

${ }^{17}$ P. Lalanne, C. Sauvan, J. P. Hugonin, J. C. Rodier, and P. Chavel, Phys. Rev. B 68, 125404 (2003).

${ }^{18}$ F. J. Garcia-Vidal, H. J. Lezec, T. W. Ebbesen, and L. MartinMoreno, Phys. Rev. Lett. 90, 213901 (2003).

${ }^{19}$ M. Sarrazin, J.-P. Vigneron, and J.-M. Vigoureux, Phys. Rev. B 67, 085415 (2003).

${ }^{20}$ Y. Xie, A. Zakharian, J. Moloney, and M. Mansuripur, Opt. Express 12, 6106 (2004).

${ }^{21}$ P. Lalanne, J. P. Hugonin, and J. C. Rodier, Phys. Rev. Lett. 95, 263902 (2005).

${ }^{22}$ Y. Xie, A. Zakharian, J. Moloney, and M. Mansuripur, Opt. Express 13, 4485 (2005).

${ }^{23}$ P. Lalanne and J. P. Hugonin, Nat. Phys. 2, 551 (2006).

${ }^{24}$ O. J. F. Martin, C. Girard, and A. Dereux, Phys. Rev. Lett. 74, 526 (1995).

${ }^{25}$ M. Paulus and O. J. F. Martin, Phys. Rev. E 63, 066615 (2001).

${ }^{26}$ C. Girard, Rep. Prog. Phys. 68, 1883 (2005). 